\title{
Detection and Quantification of Label-Free Infectious Adenovirus Using a Switch-On Cell-Based Fluorescent Biosensor
}

\author{
Miguel Ricardo Guerreiro, ${ }^{\dagger, \ddagger}$ (iD Daniela Filipa Freitas, ${ }^{\dagger, \ddagger}$ Paula Marques Alves, ${ }^{\dagger, \ddagger}$ \\ and Ana Sofia Coroadinha*,†,‡,§
}

\footnotetext{
† iBET, Instituto de Biologia Experimental e Tecnológica, Apartado 12, 2781-901 Oeiras, Portugal

‡ Instituto de Tecnologia Química e Biológica António Xavier, Universidade Nova de Lisboa, Avenida da República, 2780-157 Oeiras, Portugal

$\S$ The Discoveries Centre for Regenerative and Precision Medicine, NOVA University Lisbon, Oeiras Campus, Avenida da República, 2780-157 Oeiras, Portugal
}

\section{* Corresponding Author:}

Dr. Ana Sofia Coroadinha iBET, Instituto de Biologia Experimental e Tecnológica Apartado 12, 2781-901 Oeiras, Portugal Telephone: +351 214469457

E-mail: avalente@ibet.pt 


\section{SUPPLEMENTARY EXPERIMENTAL SECTION}

Plasmids. The coding sequence of a circular permuted Venus fluorescent protein cyclized by means of $N p u$ DnaE intein ${ }^{1}$ was designed to contain the minimal cleavable sequence LRGA $^{*} \mathrm{G}^{2}$ (asterisk denoting the scissile bond) for the AVP - backbone cVenus.v1-LRGAG - and synthesized by GenScript (Piscataway, NJ, USA). Similarly to cGFP sensor optimization, removal of codons for a methionine amino acid - cVenus.v2-LRGAG - and addition of codons for glycine amino acids surrounding the cleavable sequence - cVenus.v3-LRGAG - were performed by site-directed PCR mutation. Addition of codons for a glutamic acid and leucine amino acids to serve as a spacer for the large aromatic phenylalanine residue downstream of $\mathrm{LRGA}^{*} \mathrm{G}$ cleavable sequence ${ }^{2}$ was also evaluated - cVenus.v5-LRGAG. The coding sequence of a circular permuted mCherry fluorescent protein cyclized by means of $N p u$ DnaE intein ${ }^{1}$ was designed to contain the minimal cleavable sequence $\mathrm{LRGA}^{*} \mathrm{G}$ for AVP - backbone cCherry.v1-LRGAG - and was generated by PCR amplification from pRSET B plasmid ${ }^{3}$ of fragments coding 160-228 and 12-159 residues of mCherry. All constructions were inserted into modified pRRLSIN.cPPT.PGK-GFP.WPRE plasmids (Addgene plasmid No. 12252, kindly provided by Didier Trono through the Addgene plasmid repository, Watertown, MA, USA). Briefly, PGK-GFP was substituted by a CMV promoter driving the expression of the developed sensor sequences with an EMCV IRES, amplified from pIRESGALEO ${ }^{4}$ driving the expression of Sh ble gene conferring resistance to Zeocin, amplified from pMONO-zeo-mcs (Invivogen, San Diego, CA, USA). All described cloning reactions were conducted using In-Fusion HD Cloning system (Takara Bio Inc., Mountain View, CA, USA). Detailed sequences of the developed biosensors are shown in Table S1 and Table S2. 
Table S1: Amino acid sequences of the developed fluorescent biosensors.

\begin{tabular}{|c|c|}
\hline Name & Amino acid sequence \\
\hline \multicolumn{2}{|l|}{ superfolder-GFP variants } \\
\hline cGFP.v1-LRGAG & Dc-EF-M-Gc...AGILRGA*GELF...Gn-GS-Dn-myc \\
\hline cGFP.v2-LRGAG (cVisensor) & Dc-EF-Gc...AGILRGA*GELF...Gn-GS-Dn-myc \\
\hline cGFP.v2-IVGLG & Dc-EF-Gc...AGIIVGL*GELF...Gn-GS-Dn-myc \\
\hline cGFP.v2-MGGRG & Dc-EF-Gc...AGIMGGR*GELF...Gn-GS-Dn-myc \\
\hline cGFP.v2-IRGRG & Dc-EF-Gc...AGIIRGR*GELF...Gn-GS-Dn-myc \\
\hline cGFP.v2-NTGWG & Dc-EF-Gc...AGINTGW*GELF...Gn-GS-Dn-myc \\
\hline cGFP.v2-EEGEG & Dc-EF-Gc...AGIEEGE*GELF...Gn-GS-Dn-myc \\
\hline cGFP.v2-GIFLET & Dc-EF-Gc...AGIF*LETELF...Gn-GS-Dn-myc \\
\hline cGFP.v3-LRGAG & Dc-EF-Gc...AGIGLRGA*GGELF...Gn-GS-Dn-myc \\
\hline cGFP.v4-LRGAG & Dc-Gc...AGILRGA*GELF ...Gn-Dn-myc \\
\hline \multicolumn{2}{|l|}{ Venus variants } \\
\hline cVenus.v1-LRGAG & Dc-EF-M-Vc...AGILRGA*GFTG...Vn-GS-Dn-myc \\
\hline cVenus.v2-LRGAG & Dc-EF-Vc...AGILRGA*GFTG...Vn-GS-Dn-myc \\
\hline cVenus.v3-LRGAG & Dc-EF-Vc...AGIGLRGA*GGFTG...Vn-GS-Dn-myc \\
\hline cVenus.v5-LRGAG & Dc-EF-M-Vc...AGILRGA*GELFTG...Vn-GS-Dn-myc \\
\hline \multicolumn{2}{|l|}{ mCherry variant } \\
\hline cCherry.v1-LRGAG & Dc-EF-M-Cc...HSTLRGA*GIIK...Cn-GS-Dn-myc \\
\hline
\end{tabular}

Cleavable sequences are represented in bold, with asterisk denoting the scissile bond. Dc, Cfragment of Nostoc punctiforme DnaE intein (Npu DnaE) and CFN residues of C-extein; EF, residues coded by EcoRI endonuclease restriction site; M, methionine residue; Gc...AGI, 155-229 residues of superfolder-GFP; ELF...Gn, 6-154 residues of superfolder-GFP; Vc...AGI, 155-229 residues of Venus; FTG...Vn, 8-154 residues of Venus; Cc...HST, 160-228 residues of mCherry; IIK...Cn, 12-159 residues of mCherry; GS, residues coded by BamHI endonuclease restriction site; Dn, AEY residues of $\mathrm{N}$-extein and $\mathrm{N}$-fragment of $\mathrm{Npu}$ DnaE; myc, epitope tag derived from c-Myc protein with a GGGGS flexible linker. 
Table S2: Amino acid residues of the optimal cGFP.v2-LRGAG sensor (cVisensor).

M I K I A T R K Y L G K Q N V Y D I G V E R D H N F A L K N G F I A S N C F N

E F D K Q K N G I K A N F K I R H N V E D G S V Q L A D H Y Q Q N T P I G D

G P V L L P D N H Y L S T Q S V L S K D P N E K R D H M V L L E F V T A A G I

L R G A G E L F T G V V P I L V E L D G D V N G H K F S V R G E G E G D A T

N G K L T L K F I C T T G K L P V P W P T L V T T L T Y G V Q C F S R Y P D H

M K R H D F F K A M P E G Y Q E R T I S F K D D G T Y K T R A E V K F E

G D T L V N R I E L K G I D F K E D G N I L G H K L E Y N F N S H N V Y I T A

G S A E Y C L S Y E T E I L T V E Y G L L P I G K I V E K R I E C T V Y S V D N

N G N I T Q P VA Q W H R G E Q E F E Y C L E D G L I R A T K D K

F M T V D G Q M L I D E I F R E L D L M R V D N L P N G G G G E Q K L I

\section{S E E D L}

Amino acid residues of cGFF.v2-LRGAG (cVisensor) are color-coded as follows:

- in dark blue, C-fragment (IKI...ASN) and N-fragment (CLS...LPN) of Npu DnaE;

- in light blue, $\mathrm{C}$-extein (CFN) and N-extein (AEY) residues of $N p u$ DnaE;

- in green, 155-229 (DKQ...AGI) and 6-154 (ELF...ITA) residues of superfolder-GFP;

- in red, the LRGAG cleavable sequence;

- in grey, EF (coded by EcoRI endonuclease restriction site), GS (coded by BamHI endonuclease restriction site), and GGGGS flexible linker;

- in black, epitope tag derived from c-Myc protein. 
(A)

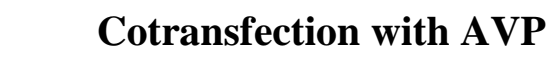

Cotransfection with mock

cGFP.v1-LRGAG
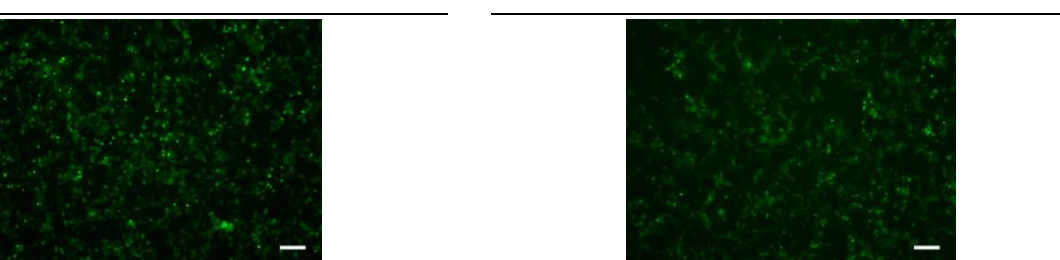

cGFP.v2-LRGAG
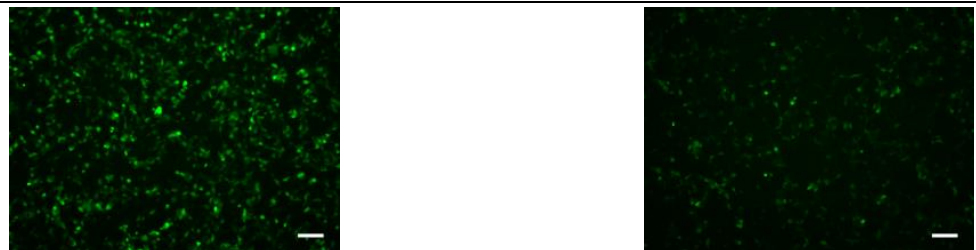

cGFP.v3-LRGAG
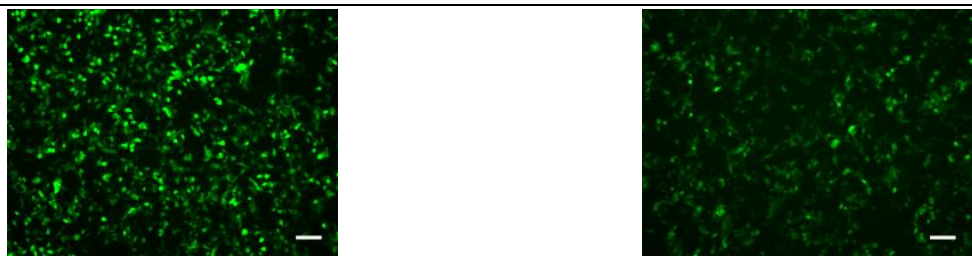

cGFP.v4-LRGAG
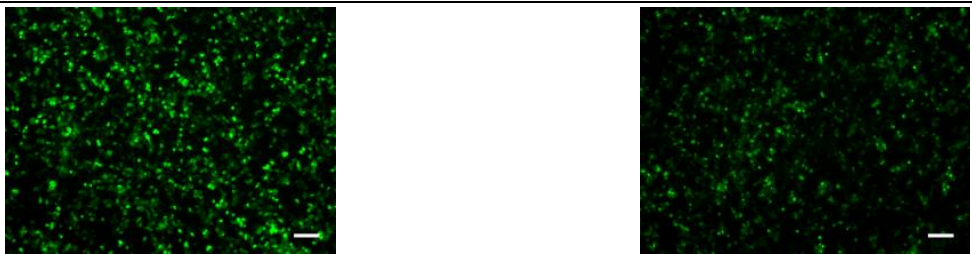

(B)
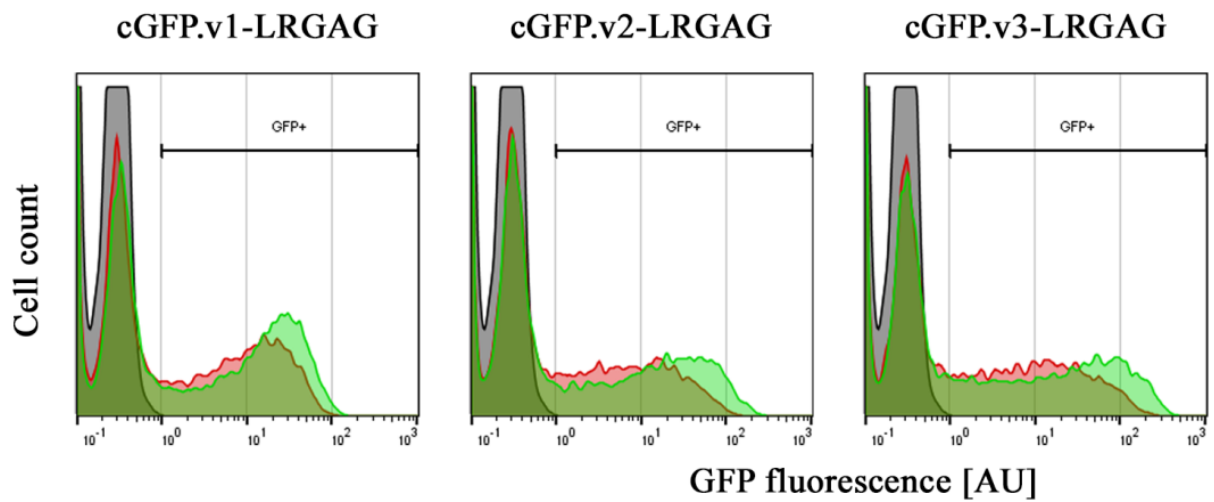

cGFP.v4-LRGAG

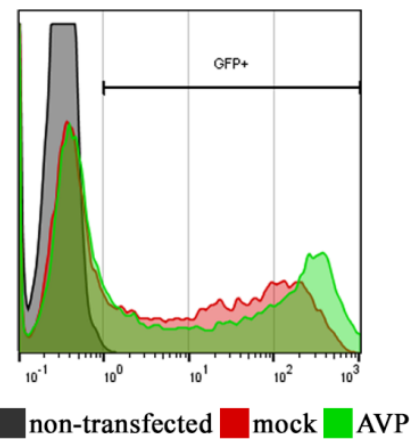

Figure S1. Evaluation of cGFP sensor's backbones. HEK-293T cells were cotransfected with plasmids coding for each of the different cGFP backbones and either adenoviral protease (AVP)coding plasmid or a mock plasmid. After $48 \mathrm{~h}$, cells were (A) visualized by fluorescence microscopy, and (B) assessed by flow cytometry for GFP fluorescence intensity (flow cytometry plots of representative experiments are shown). Scale bar $=100 \mu \mathrm{m}$. 
(A)

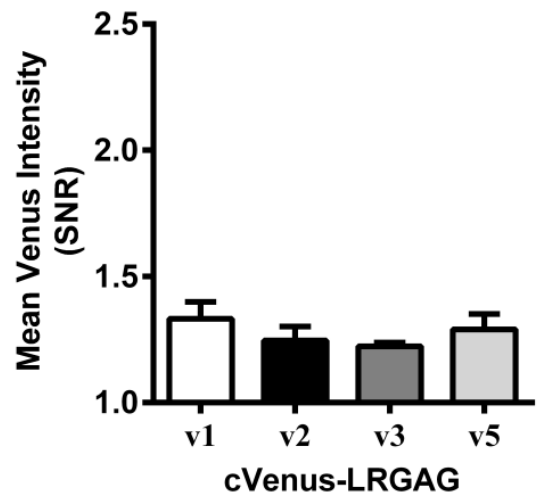

(B)

cVenus.v1-LRGAG

cVenus.v2-LRGAG

Cotransfection with AVP

Cotransfection with mock

cVenus.v3-LRGAG

cVenus.v5-LRGAG
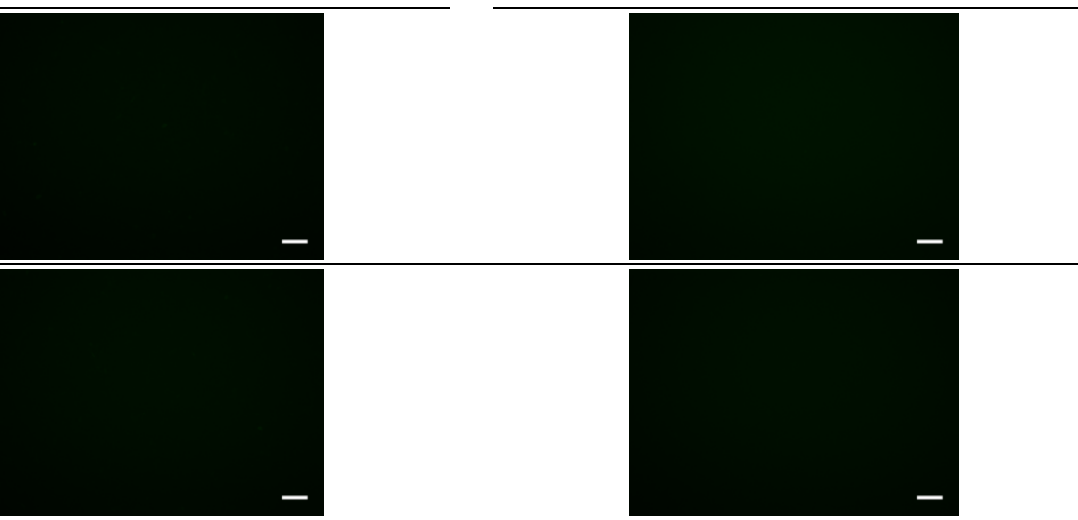

Figure S2. Evaluation of cVenus sensor's backbones. (A) HEK-293T cells were cotransfected with plasmids coding for each of the different cVenus backbones and either adenoviral protease (AVP)coding plasmid or a mock plasmid. After $48 \mathrm{~h}$, sensor performance, as given by Venus signal-tonoise ratio (SNR), was assessed by flow cytometry. Data shown as mean \pm SD of at least three independent experiments. No statistically significant differences were observed. (B) Fluorescence microscopy images of the above mentioned cotransfections. Scale bar $=100 \mu \mathrm{m}$. 
cCherry.v1-LRGAG

Cotransfection with AVP

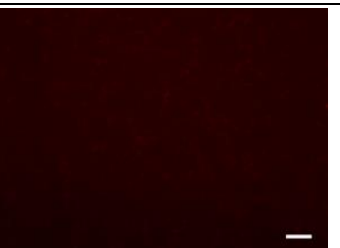

Cotransfection with mock

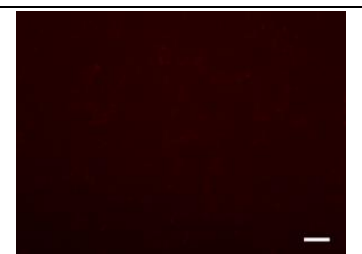

Figure S3. Evaluation of cCherry sensor. HEK-293T cells were cotransfected with plasmid coding for cCherry.v1-LRGAG and either adenoviral protease (AVP)-coding plasmid or a mock plasmid. After $48 \mathrm{~h}$, fluorescence microscopy images were acquired. Scale bar $=100 \mu \mathrm{m}$. 
(A)
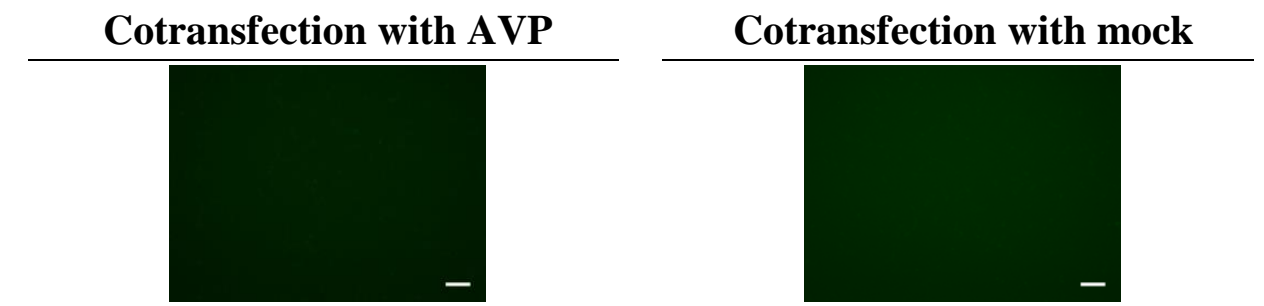

cGFP.v2-LRGAG

(cVisensor)

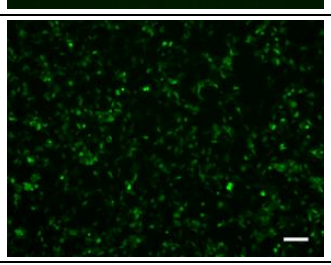

cGFP.v2-MGGRG

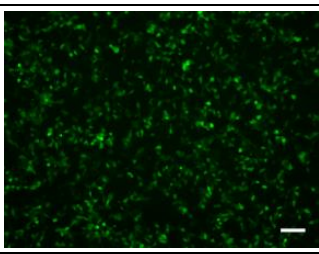

cGFP.v2-IRGRG

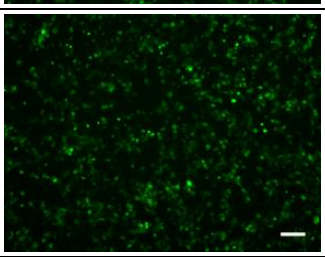

cGFP.v2-NTGWG
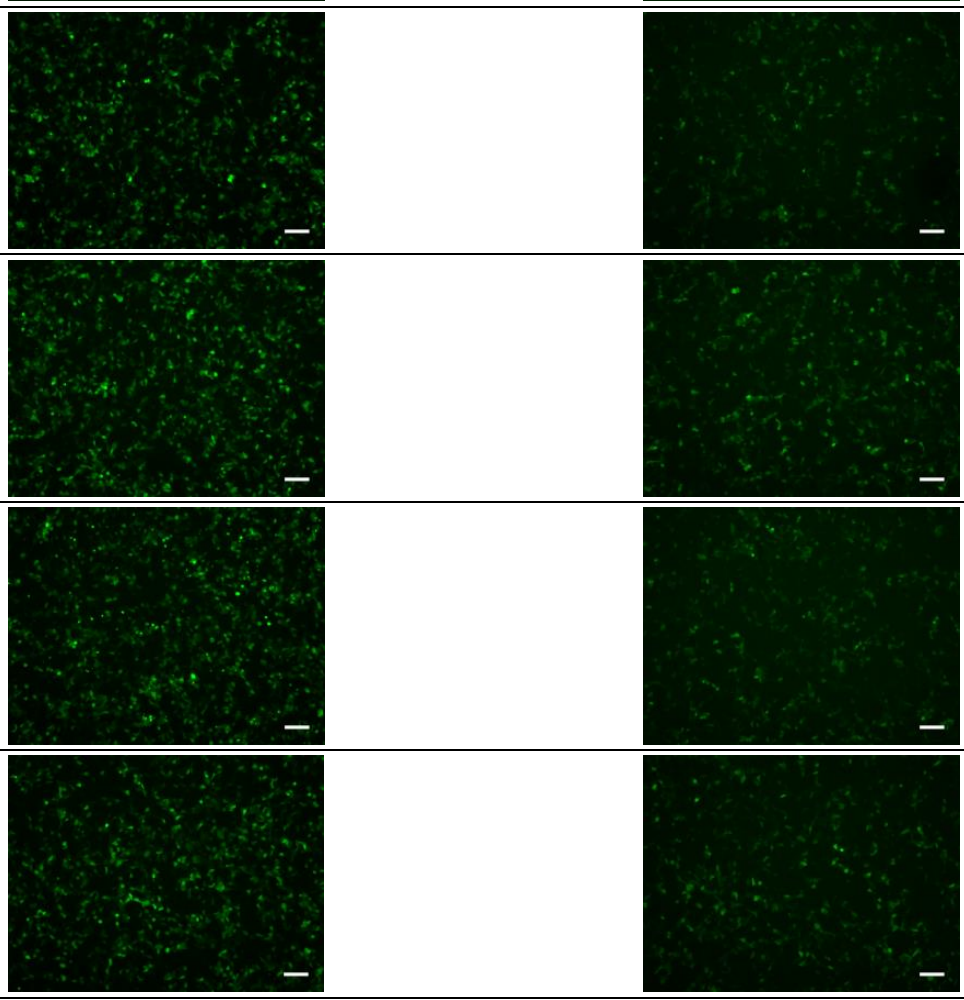

\author{
cGFP.v2-IRGRG
}

cGFP.v2-EEGEG
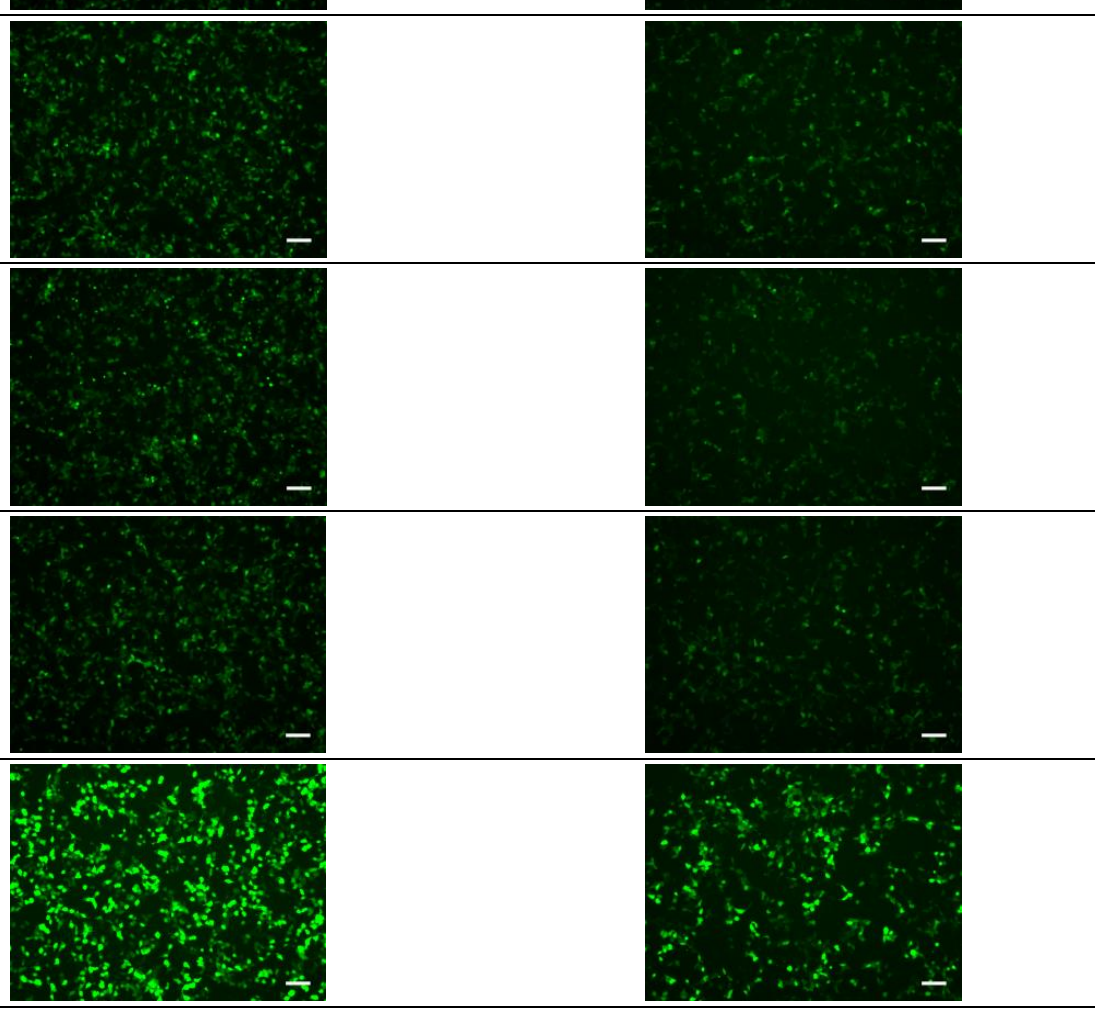

(2)

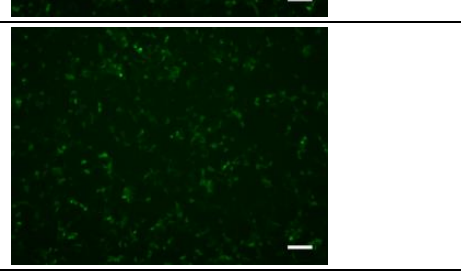

(2)

3

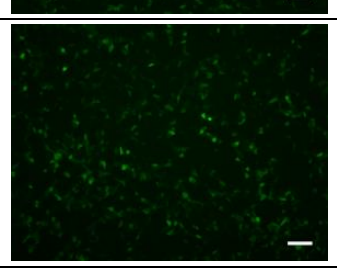

(B)

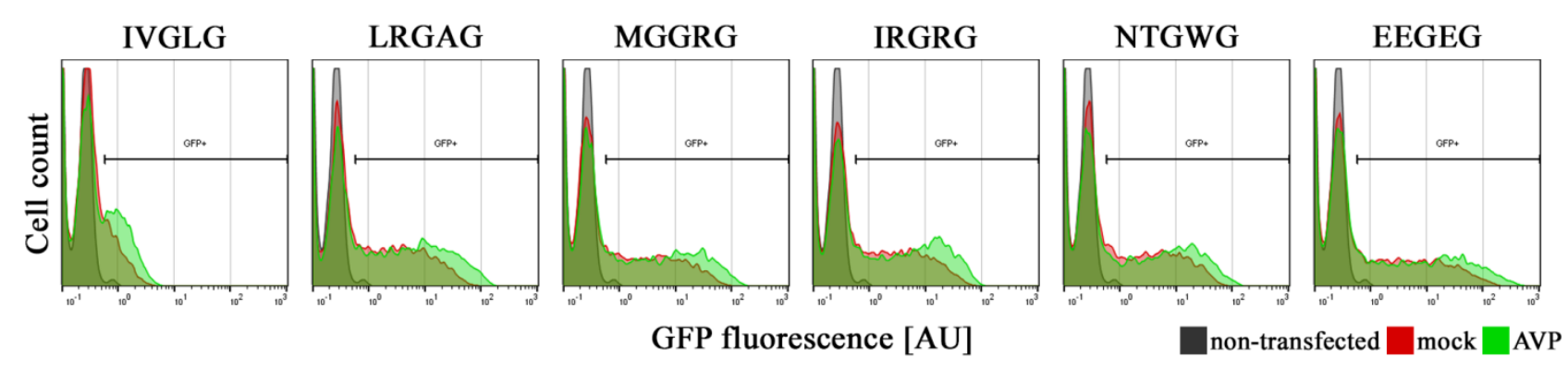

Figure S4. Evaluation of different cleavable sequences in the optimal cGFP.v2 backbone. HEK293T cells were cotransfected with plasmids coding for each of the optimal cGFP.v2 backbone with different cleavable sequences and either adenoviral protease (AVP)-coding plasmid or a mock plasmid. After 48 h, cells were (A) visualized by fluorescence microscopy, and (B) assessed by flow cytometry for GFP fluorescence intensity (flow cytometry plots of representative experiments are shown). Scale bar $=100 \mu \mathrm{m}$. 


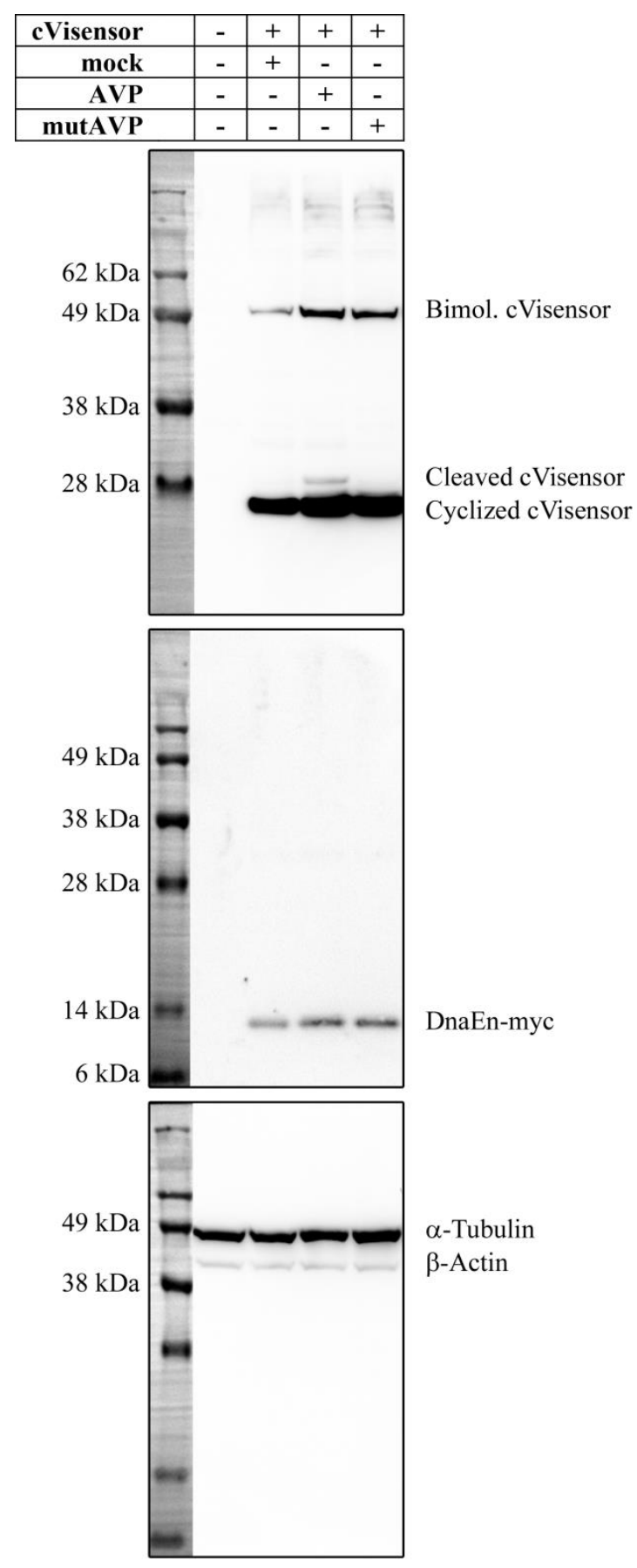

Figure S5. cVisensor validation as an adenoviral protease-dependent biosensor. HEK-293T cells were transiently cotransfected with the indicated plasmids. After $48 \mathrm{~h}$, cell extracts were generated, resolved in $12 \%(\mathrm{w} / \mathrm{v})$ SDS-PAGE gels, and analyzed by Western blotting. Overlaid images showing SeeBlue Plus2 Pre-Stained Protein Standard (Invitrogen, Carlsbad, CA, USA). Cleaved cVisensor $(27 \mathrm{kDa})$ in response to adenoviral proteolysis running slower in the gel than its cyclized counterpart $(<27 \mathrm{kDa})$, and $54 \mathrm{kDa}$ bimolecular splicing version of cVisensor (Bimol. cVisensor) were detected with anti GFP primary antibody. Release of the $13 \mathrm{kDa}$ DnaEn-myc fragment was detected with anti Myc-tag primary antibody. DnaEn-myc, myc-tagged N-fragment of Nostoc punctiforme DnaE intein. 


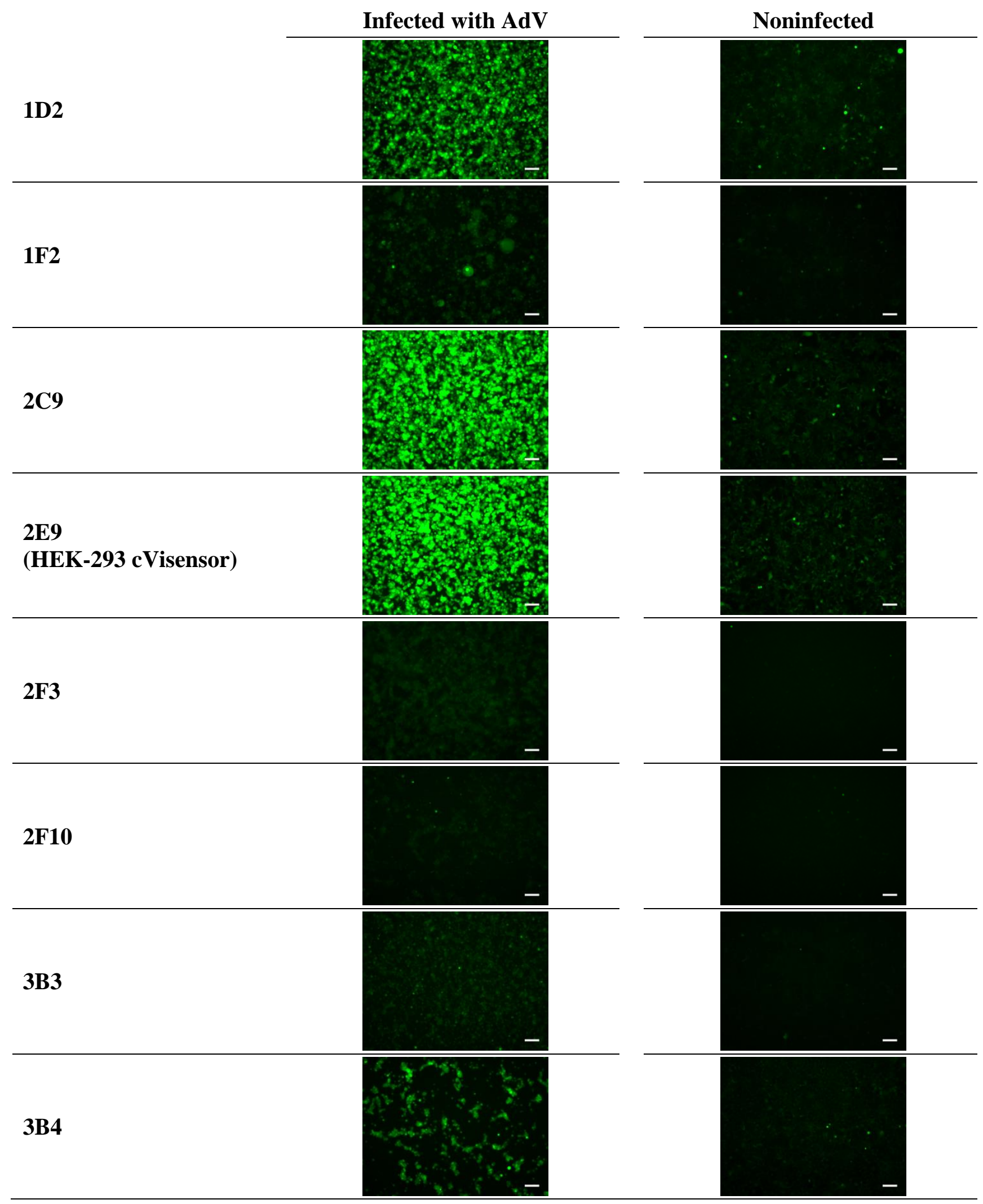

Figure S6. HEK-293 cVisensor cell clones. HEK-293 cVisensor population was cloned by limiting dilution and 27 cell clones were isolated. Eight randomly selected cell clones were infected with a characterized stock of crude lysates of adenoviral vectors (AdV) at multiplicity of infection of 5 . After $48 \mathrm{~h}$, fluorescence microscopy images were acquired. Scale bar $=100 \mu \mathrm{m}$. 


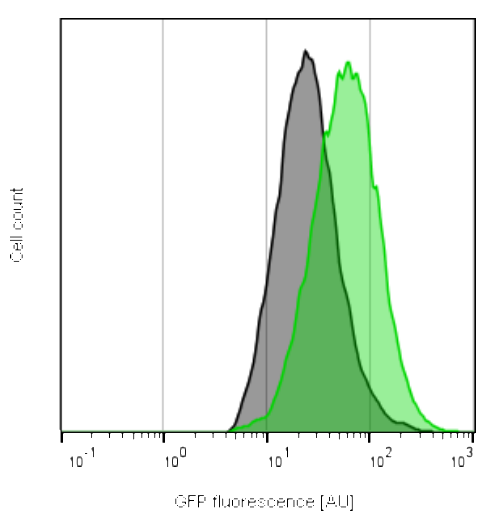

Figure S7. HEK-293 cVisensor cells in response to adenoviral infection. HEK-293 cVisensor cell clone was infected with adenoviral vectors at multiplicity of infection of 5 . After $48 \mathrm{~h}$, infected (depicted in green) and noninfected cells (in grey) were assessed by flow cytometry for GFP fluorescence intensity. Flow cytometry plot of a representative experiment is shown. 


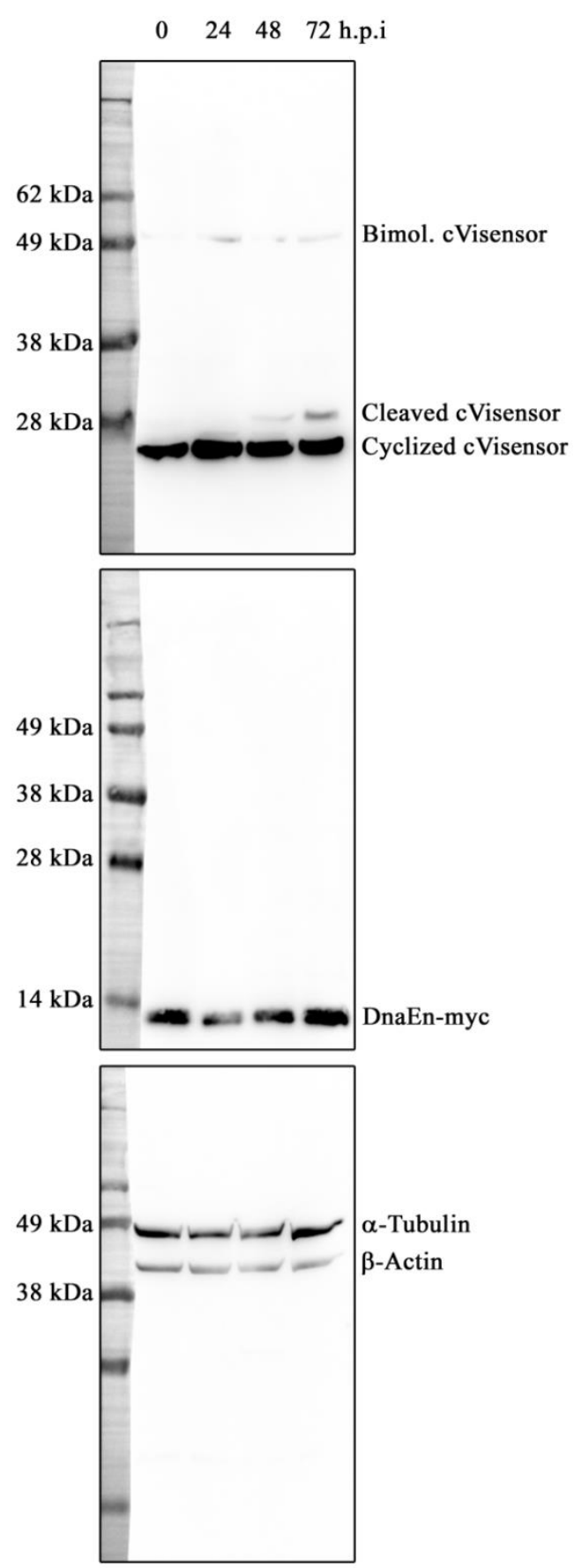

Figure S8. Live-cell monitoring of adenoviral vectors infection with cVisensor. Extracts of HEK$293 \mathrm{cVisensor}$ cell clone infected (at a multiplicity of infection of 5) with a characterized stock of purified adenoviral vectors were harvested at $0,24,48$, and $72 \mathrm{~h}$ postinfection (h.p.i.), resolved in $12 \%$ (w/v) SDS-PAGE gels, and analyzed by Western blotting. Overlaid images showing SeeBlue Plus2 Pre-Stained Protein Standard (Invitrogen). Cleaved cVisensor (27 kDa) in response to adenoviral proteolysis running slower in the gel than its cyclized counterpart $(<27 \mathrm{kDa})$, and 54 $\mathrm{kDa}$ bimolecular splicing version of cVisensor (Bimol. cVisensor) were detected with anti GFP primary antibody. Release of the $13 \mathrm{kDa}$ DnaEn-myc fragment was detected with anti Myc-tag primary antibody. DnaEn-myc, myc-tagged N-fragment of Nostoc punctiforme DnaE intein. 


\section{REFERENCES}

(1) Zhang, J.; Wang, X.; Cui, W.; Wang, W.; Zhang, H.; Liu, L.; Zhang, Z.; Li, Z.; Ying, G.; Zhang, N.; et al. Visualization of Caspase-3-like Activity in Cells Using a Genetically Encoded Fluorescent Biosensor Activated by Protein Cleavage. Nat. Commun. 2013, 4 (1), 2157. https://doi.org/10.1038/ncomms3157.

(2) Diouri, M.; Keyvani-Amineh, H.; Geoghegan, K. F.; Weber, J. M. Cleavage Efficiency by Adenovirus Protease Is Site-Dependent. J. Biol. Chem. 1996, 271 (51), 32511-32514. https://doi.org/10.1074/jbc.271.51.32511.

(3) Shaner, N. C.; Campbell, R. E.; Steinbach, P. A.; Giepmans, B. N. G.; Palmer, A. E.; Tsien, R. Y. Improved Monomeric Red, Orange and Yellow Fluorescent Proteins Derived from Discosoma Sp. Red Fluorescent Protein. Nat. Biotechnol. 2004, 22 (12), 1567-1572. https://doi.org/10.1038/nbt1037.

(4) Coroadinha, A. S.; Schucht, R.; Gama-Norton, L.; Wirth, D.; Hauser, H.; Carrondo, M. J. T. The Use of Recombinase Mediated Cassette Exchange in Retroviral Vector Producer Cell Lines: Predictability and Efficiency by Transgene Exchange. J. Biotechnol. 2006, 124 (2), 457-468. https://doi.org/10.1016/j.jbiotec.2006.01.037. 